\title{
Parecoxib has non-significant long-term effects on bone healing in rats when administered for a short period after fracture
}

\author{
Panagiotis Akritopoulos • Paraskevi Papaioannidou - Ippokratis Hatzokos • \\ Afroditi Haritanti $\cdot$ Eirini Iosifidou $\cdot$ Maria Kotoula $\cdot$ Vassiliki Mirtsou-Fidani
}

Received: 29 May 2008 / Published online: 2 August 2008

(C) The Author(s) 2008

\begin{abstract}
Introduction Selective and non-selective cyclo-oxygenase (COX) inhibitors impair bone healing by inhibiting prostaglandin synthesis. The purpose of this study was to evaluate the long-term effect of parecoxib, a selective COX-2 inhibitor, on bone healing in rats, when it is applied in a pattern similar to clinical treatment patterns, that is, in a high dose and for a short period after bone fracture.

Method Closed non-displaced mid-diaphyseal fractures in the middle of the left femoral shaft were generated in each animal. In the study group, parecoxib sodium $(1.06 \mathrm{mg} / \mathrm{kg})$ was administered intra-peritoneally every day for 7 days. In the control group, normal saline was administered intraperitoneally every day for 7 days. In both groups fracture healing (bone union and callus formation) was evaluated with X-rays 28 and 42 days after surgery.

Results Bone healing was lower in the study group (60 vs. $80 \%$ in the control group 28 days after fracture and 80 vs. $90 \% 42$ days after fracture) but this difference was not statistically significant $(P>0.05)$.
\end{abstract}

Declaration The experiments comply with the current laws of the $\mathrm{EU}$, and the protocol was approved by the Local Ethics Committee on Animal Research.

P. Akritopoulos · P. Papaioannidou $(\square) \cdot$ M. Kotoula .

V. Mirtsou-Fidani

Department of Pharmacology, Medical School,

Aristotle University of Thessaloniki, Thessaloniki, Greece

e-mail: ppap@med.auth.gr

P. Akritopoulos · I. Hatzokos · E. Iosifidou

1st Department of Orthopaedics, Medical School,

Aristotle University of Thessaloniki, Thessaloniki, Greece

A. Haritanti

Department of Radiology, Medical School,

Aristotle University of Thessaloniki, Thessaloniki, Greece
Conclusion Parecoxib does not have a significant longterm effect on bone healing in rats, when it is administered in a high dose and for a short period after bone fracture.

Keywords Parecoxib - Bone healing - Fracture healing · COX-2 inhibitors $\cdot$ COX inhibitors $\cdot$ Prostaglandins

\author{
Abbreviations \\ COX Cyclo-oxygenase \\ NSAIDs Non-steroidal anti-inflammatory drugs
}

\section{Introduction}

Non-steroidal anti-inflammatory drugs (NSAIDs) are used widely as analgesics in the treatment of acute post-operative and traumatic pain in orthopedics. NSAIDs act on cyclooxygenase (COX) and inhibit prostaglandin synthesis from arachidonic acid. Prostaglandins can stimulate bone formation as well as bone resorption, and shift the critical balance of bone resorption and formation towards formation [1,2].

By inhibiting prostaglandin synthesis, non-selective COX inhibitors can delay and decrease rates of fracture healing in treated animals [3-6]. As COX-2 plays a crucial role in fracture healing $[1,2]$, selective COX-2 inhibitors have also demonstrated inhibitory effects on bone healing. But data obtained from studies with treated animals are rather controversial. Some studies have shown that selective COX-2 inhibitors have an inhibitory effect on bone healing, similar to that of non-selective COX inhibitors [1, $7,8]$. There are also studies where COX-2 inhibitors appear to have a higher $[9,10]$ or lower or insignificant effect on bone healing [6, 11-13]. These controversies can be attributed to their transient effect on COX-2 inhibition, which is also related to dosage, gender and duration of therapy. 
The inhibitory effect of COX inhibitors on bone healing may play an important clinical role in bone rehabilitation post-operatively or after fractures, because of their wide use as analgesics in orthopedics. Because of their potentially negative effect on bone healing, several authors recommend great caution in the use of COX inhibitors and suggest to be used only for a short period after fracture or surgery $[6,14$ 20]. On the other hand, pain relief is also very important in orthopedics and it may be more substantial in achieving quick rehabilitation than real strength of bone, as it may facilitate earlier mobilization, while supporting material can be used for movement during the first post-operative days. From a clinical point of view, what seems to be more important with the use of COX inhibitors post-operatively is not their transient inhibition on bone healing but the reversibility of this inhibition and their long-term effects on bone healing and rehabilitation.

The aim of this study was to investigate radiographically the long-term effect of parecoxib, a selective COX-2 inhibitor, on fracture healing in rats, when it is administered in a pattern similar to the recommended clinical treatment patterns, that is, for a short period after fracture. The dose of parecoxib used in this study was more than threefold higher than the dose required for establishing inhibition of COX-2 [20] but quite close to doses used in clinical practice. In fact, this dose was slightly higher than the average recommended therapeutic dosages in humans, but less than twofold the highest recommended dose in clinical practice.

\section{Materials and methods}

\section{Animals}

A total of 40 adult male Albino Wistar rats, 12-week-old, weighing $250-350 \mathrm{~g}$ (mean: 295, SD: 32 ) were used in this study. The animals were obtained from the breeding colony and were housed under standard laboratory conditions (temperature $22 \pm 2^{\circ} \mathrm{C}$, humidity $50-60 \%$ and 12-h lightdark cycle). All animals were housed individually in transparent plastic cages $(24 \times 13.5 \times 13 \mathrm{~cm})$ with food and fresh tab water available ad libitum. Food was the standard rat chow, containing $1.1 \%$ calcium and $0.9 \%$ total phosphorus. The animals were divided in two groups, before operation: study group (20 animals) and control group (20 animals). The person who performed the operations was blinded to the treatment groups.

\section{Drugs}

Parecoxib sodium (dr.pd.inj. $20 \mathrm{mg}$ per vial), cefuroxime sodium (dr.pd.inj. $250 \mathrm{mg}$ per vial) and normal saline (sodium chloride inj.so.inf. 0.9\% W/V) were obtained com- mercially. Drugs were supplied in the form of an intra-peritoneal solution.

\section{Surgical procedure}

Animal research was conducted in conformity with the recommended guidelines, and the standard protocol described by Bonnarens and Einhorn [21] was used for generation of closed transverse mid-diaphyseal femoral fractures. In brief, fractures were generated by external blunt trauma to the femur after its stabilization by insertion of an intramedullary pin.

Rats were anesthetized in saturation conditions with ether. They were weighed in order to determine the dosage of the administered drugs. Appropriate depth and duration of anesthesia was achieved by intraperitoneal injections of cetamine $87 \mathrm{mg} / \mathrm{kgBW}$ and xylazine $13 \mathrm{mg} / \mathrm{kgBW}$ [22]. After confirming anesthesia, by estimating the retinal reflex, the overlying skin of the left femora was shaved and swabbed with povidone iodine for disinfection, and was draped. Through a median parapatellar skin incision, a 20gauge needle was used to ream the femoral medullary canal, through patellar tendon at the intracondular entry point. A retrograde insertion of a $0.25-\mathrm{mm}$ Kirschner wire was used for intramedullary fixation. The proximal end of the wire was isolated, cut, buried and fixed in the medullary canal. The incision was closed, and a closed mid-diaphyseal fracture was generated using a three-point bending apparatus, as described by Bonnarens and Einhorn [21]. The production of standard closed mid-diaphyseal fractures was confirmed by X-rays. The rats were monitored daily for signs of pain or distress.

Principles of laboratory animal care were followed, and Institutional guidelines for the care and treatment of laboratory animals were kept. The protocol for the use of animals was approved by the Local Ethics Committee on Animal Research.

Drug administration

In the study group parecoxib sodium $(1.06 \mathrm{mg} / \mathrm{kg})$ was administered intra-peritoneally, immediately after the surgical procedure and fracture, and once daily for 7 days. In the control group normal saline was administered intra-peritoneally, immediately after the surgical procedure and once daily for 7 days. The animals were treated with an intraperitoneal injection of cefuroxime in the prophylactic dose of $20 \mathrm{mg} / \mathrm{kg}$, $2 \mathrm{~h}$ preoperatively and once a day for the next 2 days [23].

\section{Imaging}

Plain film was chosen to confirm bone fractures and proper intramedullar fixation and to assess bone healing, as it is a 
simple and accurate method for estimating bone fracture and bone formation and healing; it is commonly used in experimental studies $[2,7]$ but it is also the most commonly used diagnostic method in clinical practice, on which usually clinical decisions are taken and rely.

Anteroposterior and lateral radiographs of all treated femora were performed immediately after fracture and at the time period of 28 and 42 days after fracture. In order to estimate evidence of delayed healing, radiographs were analyzed with respect to (1) callus formation and maturity and (2) bridging bone formation on the four cortices as seen on anteroposterior and lateral radiographs of femora in all groups of animals.

All plain films were interpreted by two independent radiologists blinded to the treatment group. The mean of duplicate scores was used for statistical analysis.

\section{Statistical analysis}

The student's $t$ test was used to determine the statistical difference between the study and the control group. Statistical difference was inferred at $P<0.05$.

\section{Results}

$\mathrm{X}$-rays performed immediately after surgery and fracture production, showed that closed transverse mid-diaphyseal fractures of the left femur were generated in all animals (Fig. 1a).

At the time period of 28 and 42 days after surgery, anteroposterior and lateral views were performed, to assess callus formation, remodeling and bridging bone formation in sagittal and coronal planes.

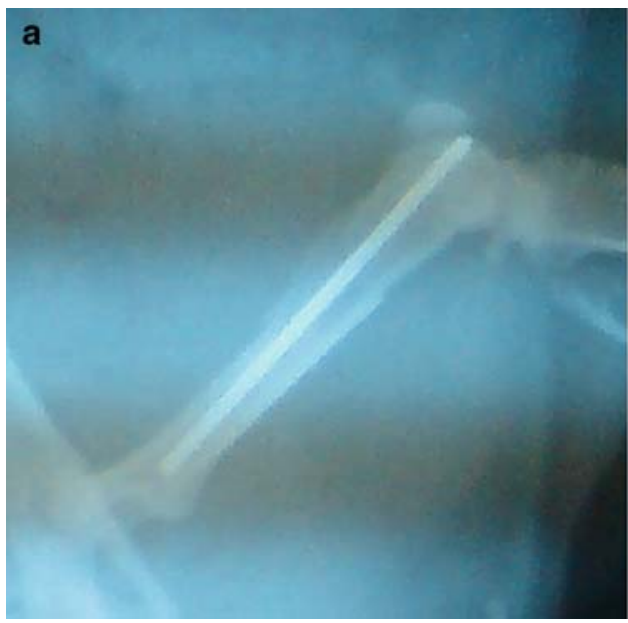

Fig. 1 Radiographic assessment of bone healing of a mid-diaphyseal fracture in an adult rat immediately after fracture and 28 days after fracture. Parecoxib sodium $(1.06 \mathrm{mg} / \mathrm{kg}$ ) was administered for 7 days immediately after fracture. a Radiographic confirmation of a closed,
Table 1 Bone healing of mid-diaphyseal fractures in adult rats 28 and 42 days after fracture

\begin{tabular}{lll}
\hline & Bone healing \\
\cline { 2 - 3 } & $\begin{array}{l}\text { 28 days after } \\
\text { fracture }\end{array}$ & $\begin{array}{l}\text { 42 days after } \\
\text { fracture }\end{array}$ \\
\hline Study group $^{\mathrm{a}}(\%)$ & $60 \%$ & $80 \%$ \\
Control group $^{\mathrm{b}}(\%)$ & $80 \%$ & $90 \%$ \\
Difference $^{\mathrm{c}}$ & $P=0.176$ & $P=0.389$
\end{tabular}

${ }^{\mathrm{a}}$ In the study group $(n=20)$ parecoxib sodium $(1.06 \mathrm{mg} / \mathrm{kg})$ was administered for 7 days immediately after fracture

b In the control group $(n=20)$ normal saline was administered for 7 days immediately after fracture

c A delay in bone healing was observed in the study group but the difference in bone healing between the study and the control group was not statistically significant $(P>0.05)$

After 28 days of fracture bone healing and bridging callus formation was confirmed by X-rays in 12 out of 20 cases $(60 \%)$ in the study group (Fig. 1b) and in 16 out of 20 cases $(80 \%)$ in the control group. This difference was not statistically significant $(P=0.176)$. After 42 days of fracture, bone healing and bridging callus formation was confirmed by X-rays in 16 out of 20 cases $(80 \%)$ and in 18 out of 20 cases $(90 \%)$, respectively. This difference was not statistically significant $(P=0.389$, Table 1$)$.

\section{Discussion}

In our study we found that parecoxib, a new selective COX-2 inhibitor, has only a transient effect and does not appear to present a significant long-term effect on bone

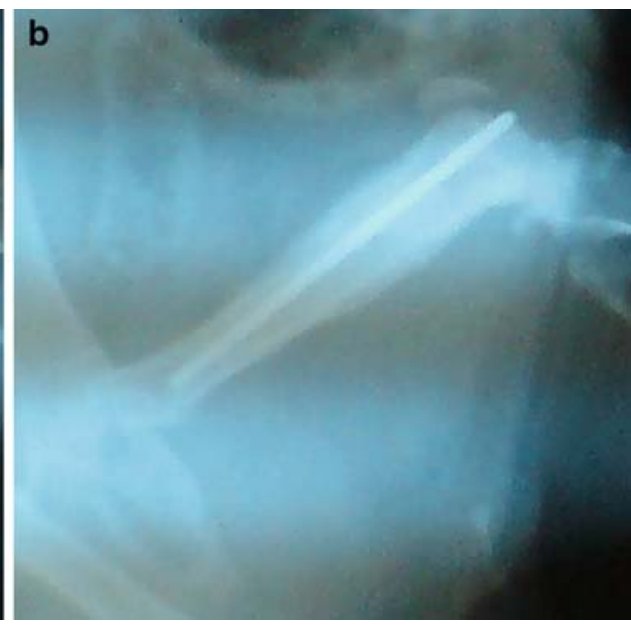

non-displaced mid-diaphyseal fracture immediately after production. Kirschner wire is fixed in the intramedullary canal. b Radiographic assessment of bone healing after 28 days. Callus formation, remodeling and bridging bone formation is observed 
healing in albino Wistar rats, when it is administered in a high dose and for a short period after fracture.

Cyclo-oxygenase inhibitors play an important role in bone healing and nociception, as they are implicated in prostaglandin synthesis. In principle, injury causes neuronal discharge of nociceptors. At the site of injury, a mixture of inflammatory substances is released and amplifies the nociceptive input by activating dormant neurons and increasing peripheral excitability [24]. Serotonin, histamine, bradycinin, cytokines, growth factors and prostaglandins are important components of this inflammatory mixture and contribute significantly to peripheral neuronal sensitization [25-27].

Bone healing is initiated by this inflammatory mixture. Two main types of cells are responsible for bone renewal: osteoblasts, involved in bone formation and osteoclasts, involved in bone resorption. The function of these cells is influenced by hormones, vitamin D and cytokines. Prostaglandins are produced by osteoblasts and act locally to modulate bone metabolism. Prostaglandins can stimulate bone formation as well as resorption. $\mathrm{PGE}_{2}$ plays a substantial role and shifts the critical balance of bone resorption and formation towards formation $[1,2]$.

Several studies have shown delayed and decreased rates of fracture healing in animals treated with non-selective COX inhibitors, like aspirin, indomethacin, naproxen, diclofenac and ketorolac [3-6, 28-32]. Nevertheless, research on selective COX-2 inhibitors has demonstrated controversial effects on bone healing: Etodolac was shown to possess an inhibitory effect on bone healing [7]. Celecoxib was found either to possess an inhibitory effect [1] or to possess a diminished or no inhibitory effect [11-13]. Studies on rofecoxib showed an inhibitory effect [1, 8], which was sometimes higher [9] and other times much lower than that observed with non-selective COX inhibitors [12]. Valdecoxib was found to have a higher inhibitory effect on bone healing, which was transient [10]. But parecoxib, which is a prodrug of valdecoxib showed a lower inhibitory effect on bone healing $[6,16]$, which was also gender dependent: it was insignificant in male and significant in female rats [16].

By inhibiting COX-2, which plays the most important role in bone formation, selective COX-2 inhibitors must present a more potent inhibition on fracture healing than non-selective COX inhibitors. This action has been demonstrated in previous works $[9,10]$ but it was also shown that it can be reversed and have minor and non important effects in a longterm period $[10,15]$. It seems also to be dependent on dosage and duration of treatment $[6,10,14,16]$. Meunier and Aspenberg [16], working with Sprague-Dawley rats showed that it is also gender dependent, and they attributed this gender-specific difference to different rates of metabolism. It is obvious that such differences are also species dependent and it is probable that they may also be race dependent.
In our study we used albino Wistar rats and observed that parecoxib, used in a high dose and for a short period after surgery, presents a transient delay but has not a significant long-term effect on bone healing. These findings are in agreement with previous works with parecoxib studied in Sprague-Dawley rats $[6,16]$, although different patterns of treatment and different approaches of interpreting data were used, and in one study [16] a different model of studying fracture healing was used. As parecoxib is a prodrug of valdecoxib, our findings are also in agreement with a previous work studying valdecoxib on Sprague-Dawley rats [10].

Currently there are no established dosage recommendations for studies in animals. And data obtained from studies in animals cannot be used directly to interpret the effect of drugs on human beings, as there are many species-dependent differences. But we believe that despite species related differences, it would be useful if dosages used in animals were close to dosages used in humans. In our study, we selected to use a dose higher than the dose required for establishing inhibition of COX-2 [20] but quite close to doses used in clinical practice. Although slightly higher than the average recommended therapeutic dosages in humans, this dose is less than twofold the highest recommended dose in clinical practice. To our knowledge, this is the first experimental work on parecoxib studied in rats, in which a treatment pattern close to current recommended clinical treatment patterns (in respect to dosage and duration of therapy) was used, and in which long-term effects of a short treatment were studied. In our study we have not investigated the initial inhibition of $\mathrm{COX}-2$, culminating in initial inhibition and delay of bone repair, as this was out of the scope of this work and as this action is not only expected theoretically but is also well documented in previous works [10, 20].

Bone fracture is a common injury, which deteriorates patients' health directly and indirectly, when rehabilitation is not optimum. In order to achieve the best possible rehabilitation, patients are encouraged for prompt mobilization but sometimes this advice is not kept because of pain. COX inhibitors are the most common pain relievers in these cases but they have the disadvantage of inhibiting and delaying bone strength and bone repair. Selective COX-2 inhibitors have been used more frequently the last years, as they exhibit analgesic and anti-inflammatory effects comparable to non-selective COX inhibitors [14, 33], while reducing the prevalence of adverse effects such as gastrointestinal bleeding and platelet abnormalities [34, 35]. But selective COX-2 inhibitors possess a potentially higher risk for inhibiting bone healing and should be used with great caution [6, 14, 17-19]. When they are used as pain relievers, and to facilitate early mobilization during the first post-operative days, patients should be advised to be very 
cautious and to use supporting orthopedic material, as bone strength is reduced by these drugs $[6,16]$; they should also be aware that they must make a short use of these drugs and stop pain relievers as soon as possible. In fact, further research is necessary, with additional experimental studies in animals and-as data on human beings are very limited-clinical trials should also be carried out, to make clear that the inhibitory action of NSAIDs on bone healing is really transient and has no irreversible effects in bone strength and bone repair.

In conclusion, our study is the first experimental study of parecoxib in rats, in which a treatment pattern close to current clinical treatment patterns was used, and in which the long-term effects of a short treatment were studied. According to our results, parecoxib, given in a high dose and for a short duration after bone trauma, seems to have a transient inhibitory effect on bone healing in rats but the long-term effects seem not to be significant. Further research is necessary to establish that its inhibitory action in bone repair is also reversible in humans, and to prove its safety when used as analgesic in bone fractures and for the relief of post traumatic and postoperative pain in orthopedics.

Conflict of interest statement The authors have no conflict of interest, and they have not received any financial support from third parties that could have an influence on this work.

Open Access This article is distributed under the terms of the Creative Commons Attribution Noncommercial License which permits any noncommercial use, distribution, and reproduction in any medium, provided the original author(s) and source are credited.

\section{References}

1. Simon A, Manigrasso M, O'Connor J (2002) Cyclo-oxygenase 2 function is essential for bone fracture healing. J Bone Miner Res 17:963-976. doi:10.1359/jbmr.2002.17.6.963

2. Zhang X, Schwarz EM, Young DA, Puzas JE, Rosier RN, O'Keefe $\mathrm{RJ}$ (2002) Cyclooxygenase-2 regulates mesenchymal cell differentiation into the osteoblasts lineage and is critically involved in bone repair. Clin Investig 109:1405-1415

3. Altman RD, Latta LL, Keer R, Renfree K, Hornicek FJ, Banovac K (1995) Effect of nonsteroidal anti-inflammatory drugs on fracture healing: a laboratory study in rats. J Orthop Trauma 9:392400. doi:10.1097/00005131-199505000-00006

4. Beck A, Krischak G, Sorg T, Augat P, Farker K, Merkel U et al (2003) Influence of diclofenac (group of nonsteroidal anti-inflammatory drugs) on fracture healing. Arch Orthop Trauma Surg 123:327-332. doi:10.1007/s00402-003-0537-5

5. Riew KD, Long J, Rhee J, Lewis S, Kuklo T, Kim YJ et al (2003) Time-dependent inhibitory effects of indomethacin on spinal fusion. J Bone Joint Surg Am 85:632-634

6. Gerstenfeld LC, Thiede M, Seibert K, Mielke C, Phippard D, Svarg B et al (2003) Differential inhibition of fracture healing by non-selective and cyclooxygenase-2 selective non-steroidal antiinflammatory drugs. J Orthop Res 21:670-675. doi:10.1016/ S0736-0266(03)00003-2
7. Endo K, Sairo K, Komatsubara S, Sasa T, Egawa H, Yonekura D et al (2002) Cyclooxygenase-2 inhibitor inhibits the fracture healing. J Physiol Anthropol 21:235-238. doi:10.2114/jpa.21.235

8. Goodman S, Ma T, Trindade M, Ikenoue T, Matsuura I, Wong N et al (2002) COX-2 selective NSAIDS decrease bone ingrowth in vivo. J Orthop Res 20:1164-1169. doi:10.1016/S0736-0266(02) 00079-7

9. Leonelli SM, Goldberg BA, Safanda J, Bagwe MR, Sethuratnam S, King SJ (2006) Effects of a cyclooxygenase-2 inhibitor (rofecoxib) on bone healing. Am J Orthop 35:79-84

10. Gerstenfeld LC, Al-Ghawas M, Alkhiary YM, Cullinane DM, Krall EA, Fitch JL et al (2007) Selective and nonselective cyclooxygenase- 2 inhibitors and experimental fracture-healing. Reversibility of effects after short-term treatment. J Bone Joint Surg Am 89:114-125. doi:10.2106/JBJS.F.00495

11. Long J, Lewis S, Kuklo T, Zhu Y, Riew KD (2002) The effect of cyclooxygenase-2 inhibitors on spinal fusion. J Bone Joint Surg Am 84:1763-1768

12. Mullins B, Copeland S, Weinhold P, et al. (2002) Effect on fracture healing in a mouse model. 48th Annual Meeting of Orthopaedic Research Society; Dallas, USA

13. Brown KM, Saunders MM, Kirsch T, Donahue HJ, Reid JS (2004) Effect of COX-2-specific inhibition on fracture-healing in the rat femur. J Bone Joint Surg Am 86:116-123

14. Gajraj NM (2003) The effect of cyclooxygenase-2 inhibitors on bone healing. Reg Anesth Pain Med 28:456-465. doi:10.1016/ j.rapm.2003.09.001

15. Gerstenfeld LC, Einhorn TA (2004) COX inhibitors and their effects on bone healing. Expert Opin Drug Saf 3:131-136. doi: $10.1517 / 14740338.3 .2 .131$

16. Meunier A, Aspenberg P (2006) Parecoxib impairs early metaphyseal bone healing in rats. Arch Orthop Trauma Surg 126:433-436. doi:10.1007/s00402-006-0160-3

17. Aspenberg P (2002) Avoid COX inhibitors after skeletal injury! Acta Orthop Scand 73:489-490. doi:10.1080/000164702321022730

18. Einhorn TA (2003) COX-2: where are we in 2003? The role of cyclooxygenase-2 in bone repair. Arthritis Res Ther 5:5-7. doi:10.1186/ar607

19. Dahners LE, Mullis BH (2004) Effects of nonsteroidal anti-inflammatory drugs on bone formation and soft tissue healing. J Am Acad Orthop Surg 12:139-143

20. FitzGerald GA, Patrono C (2001) The coxibs, selective inhibitors of cyclooxygenase. N Engl J Med 345:433-442. doi:10.1056/ NEJM200108093450607

21. Bonnarens F, Einhorn TA (1984) Production of a standard closed fracture in laboratory animal bone. J Orthop Res 2:97-101. doi:10.1002/jor.1100020115

22. Van Pelt LF (1977) Ketamine and xylazine for surgical anesthesia in rats. J Am Vet Med Assoc 171:842-844

23. Ruiz-Carretero PB, Merino-Sanjuan M, Nacher A, Casabo VG (2004) Pharmacokinetic models for the saturable absorption of cefuroxime axetil and saturable elimination of cefuroxime. Eur $\mathrm{J}$ Med Pharm Sci 21:217-223. doi:10.1016/j.ejps.2003.10.006

24. Kessler W, Kirchoff C, Reeh PW, Handwerker HO (1992) Excitation of cutaneous afferent nerve endings in vitro by a combination of inflammatory mediators and conditioning effect of substance. Exp Brain Res 91:467-476. doi:10.1007/BF00227842

25. Reuben S, Connelly N (2000) Postoperative analgesic effects of celecoxib or rofecoxib after spinal fusion surgery. Anesth Analg 91:1221-1225. doi:10.1097/00000539-200011000-00032

26. Lipsky PE (2001) The role of COX-2-specific inhibitors in clinical practice. Am J Med 110:1S-2S. doi:10.1016/S0002-9343(00) 00676-8

27. Schug SA (2006) The role of COX-2 inhibitors in the treatment of postoperative pain. Biomed Pharmacother 60:323-328. doi:10.1016/j.biopha.2006.06.017 
28. Sudmann E, Bang G (1979) Indomethacin-induced inhibition of haversian remodeling in rabbits. Acta Orthop Scand 50:621-627

29. Sudmann E, Dregelid E, Bessessen A, Morland J (1979) Inhibition of fracture healing by indomethacin in rats. Eur J Clin Invest 9:333-339. doi:10.1111/j.1365-2362.1979.tb00893.x

30. Sudmann E, Husby OS, Bang G (1982) Inhibition of partial closure of epiphyseal plate in rabbits by indomethacin. Acta Orthop Scand 53:507-511

31. Allen HL, Wase A, Bear WT (1980) Indomethacin and aspirin: effect of nonsteroidal anti-inflammatory agents on the rate of fracture repair in the rat. Acta Orthop Scand 51:595-600
32. Tornkvist H, Bauer FC, Nilson OS (1985) Influence of indomethacin on experimental bone metabolism in rats. Clin Orthop Relat Res 193:264-270

33. Seidenberg AB, Yuehuei HA (2004) Is there an inhibitory effect of COX-2 inhibitors on bone healing? Pharmacol Res 50:151-156. doi:10.1016/j.phrs.2003.12.017

34. Dannhartd G, Kiefer W (2001) Cyclooxygenase inhibitors-current status and future prospects. Eur J Med Chem 36:109-126. doi:10.1016/S0223-5234(01)01197-7

35. Hinz B, Brune K (2002) Cyclooxygenase-2 10 years later. J Pharmacol Exp Ther 300:367-375. doi:10.1124/jpet.300.2.367 